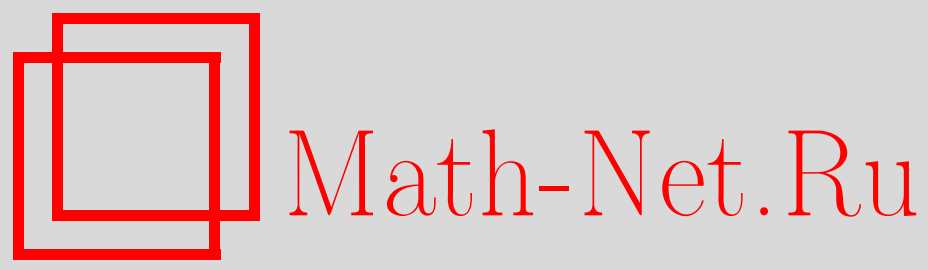

С. М. Натанзон, Пространства модулей вещественных алгебраических суперкривых с $N=2$, Функи. анализ и его прил., 1996, том 30, выпуск 4, 19-30

DOI: https://doi.org/10.4213/faa547

Использование Общероссийского математического портала MathNet.Ru подразумевает, что вы прочитали и согласны с пользовательским соглашением

http://www . mathnet.ru/rus/agreement

Параметры загрузки:

IP : 3.89 .197 .203

26 апреля 2023 г., 14:42:27

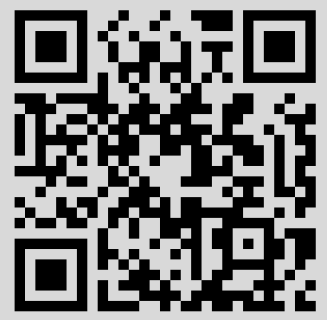


Функииональный анализ и его приложения

1996, т. 30, вып. 4, с. 19-30

УДК 515.171 .179 .8

\title{
Пространства модулей вещественных алгебраических суперкривых с $N=2$ 夫
}

\author{
(c) 1996. C. M. НАТАНЗОН
}

\section{Введение}

Суперкривые с числом нечетных координат $N=1,2$ возникли в работах $[1,2,10,11]$ как основной объект теории струн. Топологическая структура пространства модулей комплексных суперкривых и римановых суперповерхностей исследована в $[4,13]$. Пространства модулей вещественных суперкривых с $N=1$ исследованы в $[5,14]$.

В настоящей работе исследуется топологическая структура пространства модулей вещественных суперкривых с $N=2$. Найден полный набор топологических инвариантов, описывающих компоненты связности пространства модулей. Каждая компонента представлена в виде фактора $\mathbb{R}^{(m \mid n)} / \operatorname{Mod}$ линейного суперпространства по дискретной группе. Попутно описана топологическая структура пространства модулей спинорных расслоений ранга 2 (в смысле $[8,12]$ ) над вещественными алгебраическими кривыми.

\section{§1. Основные определения}

1. Пусть $L(K)$ - грассманова алгебра над полем $K$ с бесконечным числом образующих $1, e_{1}, e_{2}, \ldots$ Каждый элемент $a \in L(K)$ является конечной линейной комбинацией мономов $e_{i_{1}} \wedge \cdots \wedge e_{i_{n}}$ с коэффициентами из $K$, т. е.

$$
a=a^{\#}+\sum a_{i} e_{i}+\sum a_{i j} e_{i} \wedge e_{j}+\ldots
$$

Соответствие $a \mapsto a^{\#}$ определяет эпиморфизм \#: $L(K) \rightarrow K$.

Моном $e_{i_{1}} \wedge \cdots \wedge e_{i_{n}} \neq 0$ называется четным, если $n$ четно, и нечетным, если $n$ нечетно. Линейные комбинации четных (нечетных) мономов порождают линейное пространство $L_{0}(K)$ четных $\left(L_{1}(K)\right.$ нечетных) элементов алгебры $L(K)$. Супераналог линейного пространства - это множество

$$
K^{(n \mid m)}=\left\{\left(z_{1}, \ldots, z_{n} \mid \theta_{1}, \ldots, \theta_{m}\right): z_{i} \in L_{0}(K), \theta_{j} \in L_{1}(K)\right\} .
$$

В качестве поля $K$ будет рассматриваться поле комплексных чисел $\mathbb{C}$ или поле вещественных чисел $\mathbb{R}$.

Множество

$$
H=\left\{\left(z \mid \theta_{1}, \theta_{2}\right) \in \mathbb{C}^{(1 \mid 2)}: \operatorname{Im} z^{\#}>0\right\}
$$

* Работа выполнена при финансовой поддержке Российского фонда фундаментальных исследований (грант 95-01-01122а) и Международного научного фонда (грант MD8300). 
называется суперполуплоскостью с $N=2$. Группа $\operatorname{Aut}(H)$ его суперголомор $ф-$ ных автоморфизмов совпадает с $\operatorname{PSpO}(2 \mid 2, \mathbb{R})$ [2] и состоит из отображений $A=A[a, b, c, d, l, \varepsilon, \delta]$, таких, что

$$
\begin{aligned}
& A\left(z \mid \theta_{1}, \theta_{2}\right)=\left(\frac{a z+b+\delta^{11} \theta_{1}+\delta^{12} \theta_{2}}{c z+d+\delta^{21} \theta_{1}+\delta^{22} \theta_{2}} \mid\right. \\
& \left.\frac{l^{11} \theta_{1}+l^{12} \theta_{2}+\varepsilon^{11} z+\varepsilon^{12}}{c z+d+\delta^{21} \theta_{1}+\delta^{22} \theta_{2}}, \frac{l^{21} \theta_{1}+l^{22} \theta_{2}+\varepsilon^{21} z+\varepsilon^{22}}{c z+d+\delta^{21} \theta_{1}+\delta^{22} \theta_{2}}\right),
\end{aligned}
$$

где $a, b, c, d \in L_{0}(\mathbb{R}), l \in G L\left(2, L_{0}(\mathbb{R})\right), \varepsilon, \delta \in G L\left(2, L_{1}(\mathbb{R})\right)$,

$$
\left(\begin{array}{ll}
-c & a \\
-d & b
\end{array}\right)\left(\begin{array}{ll}
\delta^{11} & \delta^{12} \\
\delta^{21} & \delta^{22}
\end{array}\right)=\left(\begin{array}{ll}
\varepsilon^{11} & \varepsilon^{12} \\
\varepsilon^{21} & \varepsilon^{22}
\end{array}\right)\left(\begin{array}{ll}
l^{11} & l^{12} \\
l^{21} & l^{22}
\end{array}\right)
$$

$$
\begin{gathered}
a d-b c-\varepsilon^{11} \varepsilon^{22}-\varepsilon^{21} \varepsilon^{12}=l^{11} l^{22}+l^{12} l^{21}+\delta^{11} \delta^{22}+\delta^{12} \delta^{21}=\Delta, \quad \Delta^{\#}>0, \\
l^{11} l^{21}+\delta^{11} \delta^{21}=l^{12} l^{22}+\delta^{12} \delta^{22}=0 .
\end{gathered}
$$

Соответствие $\left(z \mid \theta_{1}, \theta_{2}\right) \mapsto z^{\#}$ переводит $H$ в обычную верхнюю полуплоскость $H^{\#}=\left\{z^{\#} \in \mathbb{C}: \operatorname{Im} z^{\#}>0\right\}$ и $A$ в автоморфизм $A^{\#}=\#(A) \in$ $\operatorname{Aut}\left(H^{\#}\right) \cong P S L(2, \mathbb{R})$, такой, что

$$
A^{\#} z^{\#}=\frac{a^{\#} z^{\#}+b^{\#}}{c^{\#} z^{\#}+d^{\#}} .
$$

Подгруппа $\Gamma \subset H$ называется суперфуксовой, если $\Gamma^{\#}-$ фуксова группа и ограничение \# на $\Gamma$ индуцирует изоморфизм \#: $\Gamma \rightarrow \Gamma^{\#}$. Мы рассматриваем лишь подгруппы $\Gamma$, состояшие из гиперболических элементов $\gamma \in \Gamma$, т. е. таких элементов, что $\gamma^{\#}$ имеет две неподвижные точки на $\mathbb{R}$.

В этом случае $P=H / \Gamma$ называется римановой суперповерхностью с $N=2$. Суперповерхности $P_{1}=H / \Gamma_{1}$ и $P_{2}=H / \Gamma_{2}$ считаются совпадающими, если $\Gamma_{1}=\gamma \Gamma_{2} \gamma^{-1}$, где $\gamma \in \operatorname{Aut}(H)$.

Проекция \#: $H \rightarrow H^{\#}$ индуцирует проекцию \#:P $\rightarrow P^{\#}$ на обычную риманову поверхность $P^{\#}=H^{\#} / \Gamma^{\#}$. Мы считаем, что $Г$ конечно порождена, т. е. $P^{\#}$ - поверхность рода $g$ с $n$ дырами.

Комплексной алгебраической суперкривой с $N=2$ называется риманова суперповерхность $P$ с $N=2$, такая, что $P^{\#}-$ комплексная алгебраическая кривая, т.е. $P^{\#}$ - компакт. Согласно [13], пространство модулей комплексных суперкривых с $N=2$ рода $g>1$ распадается на 6 компонент связности вида $\mathbb{R}^{(n \mid m)} / \operatorname{Mod}$, где Mod - дискретная группа. Для двух из них $(n \mid m)=(8 g-6 \mid 8 g-8)$. Для остальных $(n \mid m)=(8 g-8 \mid 8 g-8)$.

2. Напомним, что вещественной алгебраической кривой называется пара $\left(P^{\#}, \tau^{\#}\right)$, где $P^{\#}$ - комплексная алгебраическая кривая, а $\tau^{\#}: P^{\#} \rightarrow P^{\#}$ антиголоморфная инволюция. Неподвижные точки этой инволюции, т.е. точки из $P^{\tau}=\left\{p \in P^{\#}: \tau^{\#} p=p\right\}$, называются вещественными точками кривой $\left(P^{\#}, \tau^{\#}\right)$.

Две кривые $\left(P_{1}^{\#}, \tau_{1}^{\#}\right)$ и $\left(P_{2}^{\#}, \tau_{2}^{\#}\right)$ считаются совпадающими, если существует биголоморфное отображение $\varphi: P_{1}^{\#} \rightarrow P_{2}^{\#}$, такое, что $\tau_{2}^{\#}=\varphi \tau_{1}^{\#} \varphi^{-1}$. Таким образом, кривая $\left(P^{\#}, \tau^{\#}\right)$ совпадает с кривой вида $\left(H^{\#} / \Gamma^{\#}, \widetilde{\Gamma}^{\#} / \Gamma^{\#}\right)$, где 
$\widetilde{\Gamma}^{\#} \subset \widetilde{\operatorname{Aut}}\left(H^{\#}\right)-$ подгруппа группы голоморфных и антиголоморфных автоморфизмов полуплоскости $H^{\#}$ и $\Gamma^{\#}=\widetilde{\Gamma}^{\#} \cap \operatorname{Aut}\left(H^{\#}\right)$.

Супераналогом группы $\widetilde{\operatorname{Aut}}\left(H^{\#}\right)$ является группа $\widetilde{\operatorname{Aut}}(H)$, порожденная $\operatorname{Aut}(H)$ и отображением $\left(z \mid \theta_{1}, \theta_{2}\right) \mapsto\left(-\bar{z} \mid \bar{\theta}_{1}, \bar{\theta}_{2}\right)$. Пусть $\widetilde{\Gamma} \subset \widehat{\operatorname{Aut}}(H)-$ такая подгруппа, что $\Gamma=\widetilde{\Gamma} \cap \operatorname{Aut}(H)-$ суперфуксова группа, $\widetilde{\Gamma} \neq \Gamma$ и $H^{\#} / \Gamma^{\#}$ - компактная поверхность. Тогда пара $(H / \Gamma, \widetilde{\Gamma} / \Gamma)$ называется вещественной алгебраической суперкривой с $N=2$.

Вещественные суперкривые $\left(H / \Gamma_{1}, \widetilde{\Gamma}_{1} / \Gamma_{1}\right)$ и $\left(H / \Gamma_{2}, \widetilde{\Gamma}_{2} / \Gamma_{2}\right)$ считаются coвпадающими, если существует $h \in \widetilde{\operatorname{Aut}}(H)$, такой, что $\widetilde{\Gamma}_{2}=h \widetilde{\Gamma}_{1} h^{-1}$. Проекция \# переводит вещественную суперкривую $(P, \tau)=(H / \Gamma, \widetilde{\Gamma} / \Gamma)$ в вещественную кривую $\left(P^{\#}, \tau^{\#}\right)=\left(H^{\#} / \Gamma^{\#}, \widetilde{\Gamma}^{\#} / \Gamma^{\#}\right)$.

\section{§2. Топологические инварианты суперкривых}

1. Пусть $\Gamma \subset \operatorname{Aut}(H)-$ суперфуксова группа и $P=H / \Gamma-$ отвечающая ей риманова суперповерхность с $N=2$. Каждый элемент $A \in \Gamma$ сопряжен автоморфизму вида $\left(z \mid \theta_{1}, \theta_{2}\right) \mapsto\left(\lambda z \mid h^{11} \theta_{1}+h^{12} \theta_{2}, h^{21} \theta_{1}+h^{22} \theta_{2}\right)$, где $\lambda, h^{i j} \in$ $L_{0}(\mathbb{R}),\left(h^{11} h^{22}\right)^{\#} \geqslant 0,\left(h^{12} h^{21}\right)^{\#} \geqslant 0$ и или $h^{11}=h^{22}=0$, или $h^{12}=h^{21}=0$.

Определим функции $\Omega_{i}=\Omega_{i}(\Gamma): \Gamma \rightarrow \mathbb{Z}_{2}=\{0,1\}(i=1,2)$, положив

$$
\begin{aligned}
\Omega_{1}(A) & = \begin{cases}0, & \text { если } \sum_{i, j \in\{1,2\}}\left(h^{i j}\right)^{\#}<0, \\
1 & \text { в противном случае, }\end{cases} \\
\Omega_{1}(A)+\Omega_{2}(A) & = \begin{cases}0, & \text { если } h^{12}=h^{21}=0, \\
1, & \text { если } h^{11}=h^{22}=0 .\end{cases}
\end{aligned}
$$

Изоморфизмы $\Gamma \rightarrow \Gamma^{\#} \rightarrow \pi_{1}\left(P^{\#}, p\right)$ переводят $\Omega_{i}$ в $\omega_{i}=\omega_{i}(P): \pi_{1}\left(P^{\#}, p\right)$ $\rightarrow \mathbb{Z}_{2}$. Согласно [13], $\omega_{i}(a)=\omega_{i}(b)$, если $a$ и $b$ представляют один класс в $H_{1}\left(P^{\#}, \mathbb{Z}_{2}\right)$. Следовательно, $\omega_{i}$ задает функцию $\omega_{i}: H_{1}\left(P^{\#}, \mathbb{Z}_{2}\right) \rightarrow \mathbb{Z}_{2}$, причем, согласно [13], $\omega_{i}$ - функция Арфа, т. е. $\omega_{i}(a+b)=\omega_{i}(a)+\omega_{i}(b)+(a, b)$, где $(a, b) \in \mathbb{Z}_{2}$ - индекс пересечения в $H_{1}\left(P^{\#}, \mathbb{Z}_{2}\right)$.

Пусть $\left\{a_{i}, b_{i}, i=1, \ldots, g, c_{j}, j=1, \ldots, n\right\}$ - псевдосимплектический базис в $H_{1}\left(P^{\#}, \mathbb{Z}_{2}\right)$, т. е. $\left(a_{i}, a_{j}\right)=\left(b_{i}, b_{j}\right)=0,\left(a_{i}, b_{j}\right)=\delta_{i j},\left(c_{i}, a_{j}\right)=\left(c_{i}, b_{j}\right)$ $=0$. Тогда если функция Арфа $\omega: H_{1}\left(P^{\#}, \mathbb{Z}_{2}\right) \rightarrow \mathbb{Z}_{2}$ такова, что $\omega\left(c_{i}\right)=0$ $(i=1, \ldots, n)$, то четность $\delta(\omega) \in \mathbb{Z}_{2}$ суммы $\sum_{i=1}^{g} \omega\left(a_{i}\right) \omega\left(b_{i}\right)$ не зависит от выбора псевдосимплектического базиса [13]. Для удобства положим $\delta(\omega)=0$, если $\omega\left(c_{i}\right)=1$ для некоторого $i$.

2. Пусть $\left(P^{\#}, \tau^{\#}\right)$ - вещественная алгебраическая кривая рода $g$. Напомним [3], что пространство вещественных точек $P^{\tau}$ распадается на простые попарно непересекающиеся замкнутые контуры, называемые овалами. Единственными топологическими инвариантами кривой $\left(P^{\#}, \tau^{\#}\right)$ являются: 1$)$ род $g\left(P^{\#}\right)$; 2) число овалов $\left.k\left(P^{\#}, \tau^{\#}\right) ; 3\right)$ связность $\left(\varepsilon\left(P^{\#}, \tau^{\#}\right)=0\right)$ или несвязность $\left(\varepsilon\left(P^{\#}, \tau^{\#}\right)=1\right)$ множества $P^{\#} \backslash P^{\tau}$. При этом $0 \leqslant k\left(P^{\#}, \tau^{\#}\right) \leqslant g\left(P^{\#}\right)$, если $\varepsilon\left(P^{\#}, \tau^{\#}\right)=0$, и $1 \leqslant k\left(P^{\#}, \tau^{\#}\right) \leqslant g\left(P^{\#}\right)+1, k\left(P^{\#}, \tau^{\#}\right) \equiv g\left(P^{\#}\right)$ $+1(\bmod 2)$, если $\varepsilon\left(P^{\#}, \tau^{\#}\right)=1$.

Кроме овалов, на вещественной алгебраической кривой $\left(P^{\#}, \tau^{\#}\right)$ удобно рассматривать псевдоовалы, т. е. простые контуры $c \subset P^{\#} \backslash P^{\tau}$, такие, что $\tau^{\#} c=c$. 
Пусть $(P, \tau)=(H / \Gamma, \widetilde{\Gamma} / \Gamma)$ - вещественная суперкривая с $N=2$ и $C \subset \Gamma$ отвечает овалу или псевдоовалу $c$. Заменяя $\Gamma$ на сопряженную группу, можно считать, что $C\left(z \mid \theta_{1}, \theta_{2}\right)=\left(\lambda z \mid h^{1} \theta_{j}, h^{2} \theta_{3-j}\right)$. В этом случае группа $\widetilde{\Gamma}$ содержит элемент $S_{C}$ вида $S_{C}\left(z \mid \theta_{1}, \theta_{2}\right)=\left(-\rho \bar{z} \mid l^{1} \bar{\theta}_{i}, l^{2} \bar{\theta}_{3-i}\right)$, где $\rho^{\#}>0$. Положим $\mu(c)=0$, если $i=1$, и $\mu(c)=1$, если $i=2$.

Если $\omega_{1}=\omega_{2}$ (где $\left.\omega_{i}=\omega_{i}(P)\right)$, то $\mu(c)$ одинаково для всех овалов и псевдоовалов $c$, что позволяет определить инвариант $\mu(P, \tau)=\mu(c)$.

Если $\omega_{1} \neq \omega_{2}$, то ядро гомоморфизма $\left(\Omega_{1}+\Omega_{2}\right): \Gamma \rightarrow \mathbb{Z}_{2}$ образует подгруппу $\Gamma_{*}$ индекса 2. На поверхности $P_{*}^{\#}=H^{\#} / \Gamma_{*}^{\#}$ инволюции из множества $\left\{F=S_{C}\right.$ : $\mu(c)=\mu\}$ порождают инволюцию $\tau_{\mu}^{\#}\left(\mu \in \mathbb{Z}_{2}\right)$. Положим $\rho_{\mu}(P, \tau)=\varepsilon\left(P_{*}^{\#}, \tau_{\mu}^{\#}\right)$.

3. Отнесем к множеству $M(g, \varepsilon)$ вещественные алгебраические суперкривые $(P, \tau)$ с $N=2$, такие, что $g\left(P^{\#}\right)=g, \varepsilon\left(P^{\#}, \tau^{\#}\right)=\varepsilon \in \mathbb{Z}_{2}$. Структура суперкривой с $N=2$ задает две функции Арфа $\omega_{i}=\omega_{i}(P): H_{1}\left(P^{\#}, \mathbb{Z}_{2}\right) \rightarrow \mathbb{Z}_{2}$. Положим $\chi(P)=0$, если $\omega_{1}=\omega_{2}$, и $\chi(P)=1$, если $\omega_{1} \neq \omega_{2}$. Инвариант $\chi \in \mathbb{Z}_{2}$ разбивает $M(g, \varepsilon)$ на подмножества $M(g, \varepsilon, \chi)=\{(P, \tau) \in M(g, \varepsilon)$ : $\chi(P)=\chi\}$.

Согласно [7], число овалов $c \in P^{\tau}$ со свойствами $\omega_{i}(c)=0$ имеет ту же четность, что $g+1$. Для $(P, \tau) \in M(g, 0,0)$ обозначим через $k_{\alpha}(P, \tau)$ число овалов $c \subset P^{\tau}$, таких, что $\omega_{1}(c)=\omega_{2}(c)=\alpha \in \mathbb{Z}_{2}$. Разобьем множество $M(g, 0,0)$ на подмножества

$$
\begin{aligned}
& M\left(g, 0,0, k_{\alpha}, \delta, \mu\right) \\
& \quad=\left\{(P, \tau) \in M(g, 0,0): k_{\alpha}(P, \tau)=k_{\alpha}, \delta\left(\omega_{1}\right)=\delta\left(\omega_{2}\right)=\delta, \mu(P, \tau)=\mu\right\} .
\end{aligned}
$$

Для $(P, \tau) \in M(g, 0,1)$ обозначим через $k_{\alpha \beta}^{\mu}(P, \tau)$ число овалов $c \subset P^{\tau}$, таких, что

$$
\omega_{1}(c)=\alpha, \quad \omega_{2}(c)=\beta, \quad \mu(c)=\mu \in \mathbb{Z}_{2} .
$$

Положим

$$
\begin{aligned}
& M\left(g, 0,1, k_{\alpha \beta}^{\mu}, \delta_{i}, \rho_{i}\right) \\
& \quad=\left\{(P, \tau) \in M(g, 0,1): k_{\alpha \beta}^{\mu}(P, \tau)=k_{\alpha \beta}^{\mu}, \delta\left(\omega_{i}\right)=\delta_{i}, \rho_{i}(P, \tau)=\rho_{i}\right\} .
\end{aligned}
$$

Согласно [6, теорема $6.1 ; 9], M\left(g, 0,1, k_{\alpha \beta}^{\mu}, \delta_{i}, \rho_{i}\right)=0$, если $\rho_{1}=\rho_{2}=1$ или если $k_{01}^{0}+k_{10}^{0}+k_{01}^{1}+k_{10}^{1}>0$ и $\rho_{1}+\rho_{2}>0$.

4. Пусть $\left(P^{\#}, \tau^{\#}\right)$ - вещественная алгебраическая кривая, такая, что $\varepsilon\left(P^{\#}, \tau^{\#}\right)=1$, и $\omega: H_{1}\left(P^{\#}, \mathbb{Z}_{2}\right) \rightarrow \mathbb{Z}_{2}-$ функция Арфа, такая, что $\omega\left(\tau^{\#} a\right)=$ $\omega(a)$ для всех $a \in H_{1}\left(P^{\#}, \mathbb{Z}_{2}\right)$. Овалы $c_{1}, \ldots, c_{k}$ разбивают $P^{\#}$ на компоненты $P_{1}^{\#}$ и $P_{2}^{\#}$. Положим $\eta_{\omega}\left(P^{\#}, \tau^{\#}\right)=\delta\left(\omega^{\prime}\right)$, где $\omega^{\prime}$ - ограничение $\omega$ на $P_{1}^{\#}$. В частности, $\eta_{\omega}\left(P^{\#}, \tau^{\#}\right)=0$, если существует овал $c$, такой, что $\omega(c)=1$. Соединим овалы $c_{i}$ и $c_{j}$ отрезками $l_{i j}$ и $\tilde{l}_{i j} \in P_{1}^{\#}$. Положим $a_{i j}=l_{i j} \cup \tau^{\#} l_{i j}$ и $\tilde{a}_{i j}=\tilde{l}_{i j} \cup \tau^{\#} \tilde{l}_{i j}$.

ЛЕмма 2.1. $\omega\left(a_{i j}\right)=\omega\left(\tilde{a}_{i j}\right) u \omega\left(a_{i j}\right)=\omega\left(a_{i k}\right)+\omega\left(a_{k j}\right)$.

ДокАЗАтЕльство. Пусть $b \subset P_{1}^{\#}$ - замкнутый контур, состоящий из отрезков $l_{i j}, \tilde{l}_{i j}$ и частей овалов. Тогда $\omega\left(a_{i j}+\tilde{a}_{i j}\right)=\omega\left(b+\tau^{\#} b\right)=2 \omega(b)=0$, 
откуда $\omega\left(a_{i j}\right)=\omega\left(a_{i j}+\tilde{a}_{i j}\right)+\omega\left(\tilde{a}_{i j}\right)=\omega\left(\tilde{a}_{i j}\right)$. Аналогично, если $c \subset P_{1}^{\#}-$ контур, состоящий из отрезков $l_{i j}, l_{j k}, l_{k i}$ и частей овалов, то $\omega\left(a_{i j}+a_{j k}+a_{k i}\right)=$ $\omega\left(c+\tau^{\#} c\right)=2 \omega(c)=0$, откуда $\omega\left(a_{i j}\right)=\omega\left(a_{i k}\right)+\omega\left(a_{k j}\right)$.

Суперкривая $(P, \tau) \in M(g, 1)$ порождает на кривой $\left(P^{\#}, \tau^{\#}\right)$ функцию Арфа $\omega_{1}$, удовлетворяющую условиям леммы 2.1. Это позволяет корректно определить для овалов $c_{i}, c_{j} \subset P^{\tau}$ величину $\xi\left(c_{i}, c_{j}\right)=\omega_{1}\left(a_{i j}\right)$.

Для $(P, \tau) \in M(g, 1,0)$ обозначим через $k_{\alpha}^{\gamma}(P, \tau)$ число овалов $c_{i}$, таких, что $\omega_{1}\left(c_{i}\right)=\alpha$ и $\xi\left(c_{1}, c_{i}\right)=\gamma$. Набор чисел $k_{\alpha}^{\gamma}=k_{\alpha}^{\gamma}(P, \tau)$ определен с точностью до перестановки $k_{\alpha}^{\gamma} \mapsto k_{\alpha}^{1-\gamma}$, связанной с выбором $c_{1}$.

Положим

$$
\begin{aligned}
& M\left(g, 1,0, k_{\alpha}^{\gamma}, \eta, \mu\right) \\
& \quad=\left\{(P, \tau) \in M(g, 1,0): k_{\alpha}^{\gamma}(P, \tau)=k_{\alpha}^{\gamma}, \eta_{\omega_{1}}(P, \tau)=\eta, \mu(P, \tau)=\mu\right\} .
\end{aligned}
$$

Пусть теперь $(P, \tau) \in M(g, 1,1)$. Обозначим через $k_{\alpha \beta}^{\gamma \mu}(P, \tau)$ число овалов $c_{i} \subset P^{\tau}$, таких, что $\omega_{1}\left(c_{i}\right)=\alpha, \omega_{2}\left(c_{i}\right)=\beta, \xi\left(c_{1}, c_{i}\right)=\gamma$ и $\mu\left(c_{i}\right)=\mu$. Набор чисел $k_{\alpha \beta}^{\gamma \mu}=k_{\alpha \beta}^{\gamma \mu}(P, \tau)$ определен с точностью до перестановки $k_{\alpha \beta}^{\gamma \mu} \mapsto k_{\alpha \beta}^{1-\gamma \mu}$, связанной с выбором $c_{1}$. Положим

$$
M\left(g, 1,1, k_{\alpha \beta}^{\gamma \mu}, \eta_{i}\right)=\left\{(P, \tau) \in M(g, 1,1): k_{\alpha \beta}^{\gamma \mu}(P, \tau)=k_{\alpha \beta}^{\gamma \mu}, \eta_{\omega_{i}}(P, \tau)=\eta_{i}\right\} .
$$

Таким образом, мы получили следующий результат:

Теорема 2.1. (1) Множество $M(g, \varepsilon, 0)$ вещественных суперкривых $(P, \tau)$ рода $g$, таких, ито $\omega_{1}(P)=\omega_{2}(P)$, распадается на подмножества $M\left(g, 0,0, k_{\alpha}, \delta, \mu\right), M\left(g, 1,0, k_{\alpha}^{\gamma}, \eta, \mu\right)$, где $\alpha, \gamma, \delta, \eta, \mu \in \mathbb{Z}_{2}, 0 \leqslant k_{0}+k_{1} \leqslant g$, $1 \leqslant \sum_{\alpha, \gamma} k_{\alpha}^{\gamma} \leqslant g+1, \sum_{\alpha, \gamma} k_{\alpha}^{\gamma} \equiv g+1(\bmod 2), k_{0} \equiv g+1(\bmod 2), k_{0}^{0}+k_{0}^{1} \equiv$ $g+1(\bmod 2)$ и $\eta=0$ при $k_{1}^{0}+k_{1}^{1}>0$. Среди них совпадают лишь множества $M\left(g, 1,0, k_{\alpha}^{\gamma}, \eta, \mu\right) u M\left(g, 1,0, k_{\alpha}^{1-\gamma}, \eta, \mu\right)$.

(2) Множество $M(g, \varepsilon, 1)$ вещественных суперкривых $(P, \tau)$ рода $g$, та-

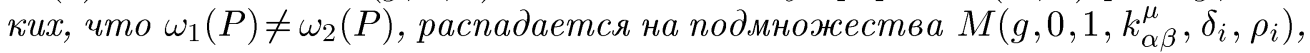
$M\left(g, 1,1, k_{\alpha \beta}^{\gamma \mu}, \eta_{i}\right)$, где $\alpha, \beta, \gamma, \mu, i, \delta_{i}, \rho_{i}, \eta_{i} \in \mathbb{Z}_{2}, 0 \leqslant \sum_{\alpha, \beta, \mu} k_{\alpha \beta}^{\mu} \leqslant g, 1 \leqslant$ $\sum_{\alpha, \beta, \gamma, \mu} k_{\alpha \beta}^{\gamma \mu} \leqslant g+1, \sum_{\alpha, \beta, \gamma, \mu} k_{\alpha \beta}^{\gamma \mu} \equiv g+1(\bmod 2), \sum_{\mu, \beta} k_{0 \beta}^{\mu} \equiv \sum_{\mu, \alpha} k_{\alpha 0}^{\mu} \equiv$ $\sum_{\gamma, \mu, \beta} k_{0 \beta}^{\gamma \mu} \equiv \sum_{\gamma, \mu, \alpha} k_{\alpha 0}^{\gamma \mu} \equiv g+1(\bmod 2), \rho_{1}+\rho_{2}<2, \rho_{1}=\rho_{2}=0$ npu $k_{01}^{0}+k_{01}^{1}+$ $k_{10}^{0}+k_{10}^{1}>0, \eta_{1}=0$ nрu $\sum_{\alpha, \gamma, \mu} k_{1 \beta}^{\gamma \mu}>0$ u $\eta_{2}=0$ nрu $\sum_{\alpha, \gamma, \mu} k_{\alpha 1}^{\gamma \mu}>0$. Cредu них совпадают лишь множества $M\left(g, 1,1, k_{\alpha \beta}^{\gamma \mu}, \eta_{i}\right)$ u $M\left(g, 1,1, k_{\alpha \beta}^{1-\gamma \mu}, \eta_{i}\right)$.

ЗАмЕЧАНИЕ 1 . Ниже мы покажем, что каждому из множеств, описанных в теореме 2.1, отвечает компонента связности пространства модулей вещественных суперкривых с $N=2$ и, следовательно, набор описанных инвариантов полон. Каждой компоненте связности отвечает топологический тип неособой пары функций Арфа в смысле [7]. Все такие типы представлены одним или двумя множествами. Совпадающий тип имеют пары

$$
\begin{gathered}
\left(M\left(g, 1,0, k_{\alpha}^{\gamma}, \eta, 0\right), M\left(g, 1,0, k_{\alpha}^{\gamma}, \eta, 1\right)\right), \\
\left(M\left(g, 1,0, k_{\alpha \beta}^{\mu}, \delta_{i}, \rho_{i}\right), M\left(g, 1,0, k_{\alpha \beta}^{1-\mu}, \delta_{i}, \rho_{i}\right)\right), \\
\left(M\left(g, 1,1, k_{\alpha \beta}^{\gamma \mu}, \delta_{i}\right), M\left(g, 1,1, k_{\alpha \beta}^{\gamma 1-\mu}, \delta_{i}\right)\right) .
\end{gathered}
$$


Отметим в связи с этим неточность в теореме 4.4 из [7]: при $k_{01}+k_{10}>0$ инвариант $k_{\alpha \beta}$ необходимо разбить на неупорядоченную пару инвариантов $k_{\alpha \beta}^{\mu}$ $\left(\mu \in \mathbb{Z}_{2}\right)$.

ЗАмЕчАние 2. Сопоставим автоморфизму $A=A[a, b, c, l, \varepsilon, \delta] \in \operatorname{Aut}(H)$ автоморфизм

$$
A^{*}\left(z \mid \theta_{1}, \theta_{2}\right)=\left(\frac{a^{\#} z+b^{\#}}{c^{\#} z+d^{\#}} \mid \frac{\left(l^{11}\right)^{\#} \theta_{1}+\left(l^{12}\right)^{\#} \theta_{2}}{\left(c^{\#} z+d^{\#}\right)^{-1}}, \frac{\left(l^{21}\right)^{\#} \theta_{1}+\left(l^{22}\right)^{\#} \theta_{2}}{\left(c^{\#} z+d^{\#}\right)^{-1}}\right)
$$

множества $H^{*}=H \times C^{2}$. Соответствие $*$ переводит комплексную суперкривую $P$ с $N=2$ в спинорное расслоение ранга 2 над комплексной кривой (в смысле $[8,12])$ и вещественную суперкривую $(P, \tau)$ с $N=2$ в вещественное спинорное расслоение ранга 2 над вещественной кривой. Таким образом, описание компонент пространства модулей вещественных кривых дает описание компонент связности пространства модулей спинорных расслоений ранга 2 над вещественными кривыми.

\section{§3. Параметризация компонент связности пространства модулей кривых $(\boldsymbol{P}, \tau)$, удовлетворяющих условию $\omega_{1}(\boldsymbol{P})=\omega_{2}(\boldsymbol{P})$}

1. Пусть $C \subset \operatorname{Aut}(H)$ - гиперболический автоморфизм и $F=F_{C} \in \operatorname{Aut}(H)$ таков, что $F^{-1} C F\left(z \mid \theta_{1}, \theta_{2}\right)=\left(\lambda z \mid h^{1} \theta_{i}, h^{2} \theta_{3-i}\right)$ и $\lambda^{\#}>1$. Положим $S_{C}^{\gamma \mu}=$ $F S^{\gamma \mu} F^{-1}$, где $S^{00}\left(z \mid \theta_{1}, \theta_{2}\right)=\left(-\bar{z} \mid \bar{\theta}_{1}, \bar{\theta}_{2}\right), \quad S^{01}\left(z \mid \theta_{1}, \theta_{2}\right)=\left(-\bar{z} \mid \bar{\theta}_{2}, \bar{\theta}_{1}\right)$, $S^{10}\left(z \mid \theta_{1}, \theta_{2}\right)=\left(-\bar{z} \mid-\bar{\theta}_{1},-\bar{\theta}_{2}\right)$ и $S^{11}\left(z \mid \theta_{1}, \theta_{2}\right)=\left(-\bar{z} \mid-\bar{\theta}_{2},-\bar{\theta}_{1}\right)$.

Положим $\widetilde{S}_{C}^{\gamma \mu}=F I_{C} S^{\gamma \mu} F^{-1}$, где $I_{C}\left(z \mid \theta_{1}, \theta_{2}\right)=\left(\sqrt{\lambda} z \mid \theta_{1}, \theta_{2}\right)$. Проекция \# переводит $S_{C}^{\gamma \mu}$ в $S_{C}^{\#}=F^{\#} S^{\#}\left(F^{\#}\right)^{-1}$ и $\widetilde{S}_{C}^{\gamma \mu}$ в $\widetilde{S}_{C}^{\#}=F^{\#}\left(I_{C}\right)^{\#} S^{\#}\left(F^{\#}\right)^{-1}$, где $S^{\#}(z)=-\bar{z}$.

Пусть $(H / \Gamma, \widetilde{\Gamma} / \Gamma)$ - вещественная суперкривая, $C \in \Gamma$ и $I=\left\{z \in H^{\#}\right.$ : $\operatorname{Re} z=0\}$. Естественная проекция $H^{\#} \rightarrow P^{\#}=H^{\#} / \Gamma^{\#}$ переводит $F(I)$ в отвечающий $C$ контур $c \subset P^{\#}$. Ориентация $F_{C}^{-1} C F_{C}=I$ по возрастанию $\operatorname{Im} z$ индуцирует ориентацию на $c$, которую мы назовем ориентацией, порожденной $C$. Если $c$ - овал (псевдоовал), то существует ровно одна пара $\gamma, \mu \in \mathbb{Z}_{2}$, такая, что $S_{C}^{\gamma \mu} \subset \widetilde{\Gamma}\left(\widetilde{S}_{C}^{\gamma \mu} \subset \widetilde{\Gamma}\right)$. Зададим на $c$ еще одну ориентацию (мы назовем ее спинорной), которая совпадает с ориентацией, порожденной $C$, если $\gamma=0$, и противоположна ей, если $\gamma=1$.

ЛЕмма 3.1 [5]. Пусть сдвиги $C_{1}, C_{2} \subset \Gamma$ отвечают овалам или псевдоовалам $c_{1}, c_{2}$ суперкривой $(P, \tau)=(H / \Gamma, \widetilde{\Gamma} / \Gamma)$ и сдвигу $C_{1} \cdot C_{2}$ отвечает простой контур $с \subset P^{\#}$. Пусть $S_{i}=S_{C_{i}}^{\gamma_{i} \mu_{i}} \in \widetilde{\Gamma}$, если $c_{i}-$ овал, и $S_{i}=\widetilde{S}_{C_{i}}^{\gamma_{i} \mu_{i}} \in \widetilde{\Gamma}$, если $c_{i}$ - псевдоовал. Тогда $\Omega_{1}(\Gamma)\left(S_{1} S_{2}\right)=\gamma_{1}+\gamma_{2}$.

2. Введем множество $T\left(g_{0}, n_{\alpha}, \eta\right)\left(\right.$ где $\alpha, \eta \in \mathbb{Z}_{2}, n_{1} \equiv 0(\bmod 2), n=$ $n_{0}+n_{1}>0$ и $\eta=0$ при $\left.n_{1}>0\right)$. Оно состоит из всех наборов $t=\left\{A_{i}, B_{i}(i=\right.$ $\left.\left.1, \ldots, g_{0}\right), C_{j}(j=1, \ldots, n)\right\} \subset(\operatorname{Aut}(H))^{g+1}$, таких, что

1) $C_{1}\left(z \mid \theta_{1}, \theta_{2}\right)=\left(\lambda z \mid h^{1} \theta_{1}, h^{2} \theta_{2}\right)$, где $\lambda^{\#}>1$;

2) $C_{n}(1 \mid 0,0)=(1 \mid 0,0)$, если $n>1$, и $A_{1}(1 \mid 0,0)=(1 \mid 0,0)$, если $n=1$, причем в первом случае $1 \in \mathbb{R}$ - притягивающая точка для $C_{1}^{\#}$, а во втором - для $A_{1}^{\#}$; 
3) набор $t$ порождает суперфуксову группу Г с определяющим соотношением

$$
\prod_{i=1}^{g_{0}}\left[A_{i} B_{i}\right] \prod_{j=1}^{n} C_{j}=1
$$

состоящую из гиперболических сдвигов, сопряженных сдвигам $\left(z \mid \theta_{1}, \theta_{2}\right) \mapsto$ $\left(\lambda z \mid h^{1} \theta_{1}, h^{2} \theta_{2}\right)$ (т.е. $\left.\Omega_{1}(\Gamma)=\Omega_{2}(\Gamma)\right)$;

4) на поверхности $P^{\#}=H^{\#} / \Gamma^{\#}$ (рода $g_{0}$ с $n$ дырами) сдвигам $C_{i}$ отвечают простые контуры, охватывающие ровно одну дыру, причем

$$
\Omega\left(C_{i}\right)= \begin{cases}0 & \text { при } i \leqslant n_{0}, \\ 1 & \text { при } i>n_{0}\end{cases}
$$

где $\Omega=\Omega_{1}(\Gamma)=\Omega_{2}(\Gamma)$;

5) $\Omega\left(A_{i}\right)=\Omega\left(B_{i}\right)=0$ при $i>1$ и $\Omega\left(A_{1}\right)=\Omega\left(B_{1}\right)=\eta$.

Лемма 3.2. Рассмотрим отображение $\Phi=\Phi\left(k_{\alpha}^{\gamma}, \mu\right)$ (где $\alpha, \gamma, \mu \in \mathbb{Z}_{2}$, $\left.k_{\alpha}^{0}+k_{\alpha}^{1}=n_{\alpha}\right)$, сопоставляюшее набору $t=\left\{A_{i}, B_{i}\left(i=1, \ldots, g_{0}\right), C_{j}(j=\right.$ $1, \ldots, n)\} \in T\left(g_{0}, n_{\alpha}, \eta\right)$ кривую $(P, \tau)=(H / \Gamma, \widetilde{\Gamma} / \Gamma)$, где группа $\widetilde{\Gamma}$ nорождена набором $t$ и инволючиями $S_{C_{i}}^{\gamma_{i} \mu}$, такими, что

$$
\gamma_{i}= \begin{cases}0 & \text { при } i \leqslant k_{0}^{0} \text { или } n_{0}<i \leqslant n_{0}+k_{1}^{0}, \\ 1 & \text { при } k_{0}^{0}<i \leqslant n_{0} \quad \text { или } i>n_{0}+k_{1}^{0} .\end{cases}
$$

Тогда $\Phi\left(T\left(g_{0}, n_{\alpha}, \eta\right)\right)=M\left(g, 1,0, k_{\alpha}^{\gamma}, \eta, \mu\right)$, где $g=2 g_{0}+n-1$.

ДокаЗАТЕльство. Утверждение $\Phi\left(T\left(g_{0}, n_{\alpha}, \eta\right)\right) \subset M\left(g, 1,0, k_{\alpha}^{\gamma}, \eta, \mu\right)$ следует из леммы 3.1. Пусть $(P, \tau) \in M\left(g, 1,0, k_{\alpha}^{\gamma}, \eta, \mu\right)$. Занумеруем овалы $c_{1}, \ldots, c_{n}$ кривой $\left(P^{\#}, \tau^{\#}\right)$ так, чтобы

$$
\omega\left(c_{i}\right)= \begin{cases}0 & \text { при } i \leqslant n_{0}, \\ 1 & \text { при } i>n_{0}\end{cases}
$$

и

$$
\xi^{\omega}\left(c_{1}, c_{i}\right)= \begin{cases}0 & \text { при } i \leqslant k_{0}^{0} \text { или } n_{0}<i<n_{0}+k_{1}^{0}, \\ 1 & \text { при } k_{i}^{0}<i \leqslant n_{0} \text { или } i>n_{0}+k_{1}^{0},\end{cases}
$$

где $\omega=\omega_{1}(P)=\omega_{2}(P)$. Наделим контур $c_{1}$ ориентацией, совпадающей со спинорной, если $\gamma_{1}=0$, и противоположной ей, если $\gamma_{1}=1$. Согласно [3], кривая $\left(P^{\#}, \tau^{\#}\right)=\left(H^{\#} / \Gamma^{*}, \widetilde{\Gamma}^{*} / \Gamma^{*}\right)$, где $\widetilde{\Gamma}^{*} \subset \widetilde{\operatorname{Aut}}\left(H^{\#}\right)$, порождена множеством $t^{*}=$ $\left\{A_{i}^{*}, B_{i}^{*}\left(i=1, \ldots, g_{0}\right), C_{j}^{*}(j=1, \ldots, n)\right\} \subset T\left(g_{0}, n_{\alpha}, \eta\right)$ и инволюциями $S_{C_{i}^{*}}^{\#}$, где сдвиги $C_{j}^{*}$ отвечают овалам $c_{j}$ и ориентация $c_{1}$ индуцирована $C_{1}$.

Выберем $\widetilde{\Gamma} \subset \widetilde{\operatorname{Aut}}(H)$ так, чтобы $(P, \tau)=(H / \Gamma, \widetilde{\Gamma} / \Gamma)$ и $\widetilde{\Gamma}^{\#}=\widetilde{\Gamma}^{*}$. Положим $t=(\#)^{-1}\left(t^{*}\right) \subset \Gamma$. Заменяя $t$ на сопряженный, можно считать, что $t=\left\{A_{i}, B_{i}\left(i=1, \ldots, g_{0}\right), C_{j}(j=1, \ldots, n)\right\}$ удовлетворяет условиям 1$\left.)-4\right)$ разд. 2 и $S_{C_{1}}^{\gamma_{1} \mu} \subset \widetilde{\Gamma}$. Согласно [13], $\left\{A_{i}^{*}, B_{i}^{*}\left(i=1, \ldots, g_{0}\right)\right\}$ можно выбрать таким образом, чтобы выполнялись условия 5), т. е. $t \subset T\left(g_{0}, n_{\alpha}, \eta\right)$. Согласно лемме 3.1, $S_{C_{i}}^{\gamma_{i} \mu} \subset \widetilde{\Gamma}$ при всех $i$ и, следовательно, $\Phi(t)=(P, \tau)$.

Лемма 3.3. Рассмотрим отображение $\Phi=\Phi\left(k_{\alpha}, \delta, \mu\right)$ (где $\alpha, \delta, \mu \in$ $\left.\mathbb{Z}_{2}, k_{0}=n_{0}, n_{1}-2 \leqslant k_{1}<n_{1}\right)$, сопоставляющее набору $t=\left\{A_{i}, B_{i}(i=\right.$ 
$\left.\left.1, \ldots, g_{0}\right), C_{\tilde{\widetilde{\Gamma}}}(j=1, \ldots, n)\right\} \in T\left(g_{0}, n_{\alpha}, 0\right)$ кривую $(P, \tau)=(H / \Gamma, \widetilde{\Gamma} / \Gamma)$, где группа $\widetilde{\Gamma}$ порождена набором $t$ и преобразованиями $S_{C_{i}}^{0 \mu}\left(i<k_{0}+k_{1}\right)$, $\widetilde{S}_{C_{i}}^{0 \mu}\left(k_{0}+k_{1}<i<n\right), S_{C_{n}}^{\delta \mu}$. Тогда $\Phi\left(T\left(g_{0}, n_{\alpha}, 0\right)\right)=M\left(g, 0,0, k_{\alpha}, \delta, \mu\right)$, где $g=2 g_{0}+n-1$.

ДокаЗАТЕЛЬСтво. Утверждение $\Phi\left(T\left(g_{0}, n_{\alpha}, 0\right)\right) \subset M\left(g, 0,0, k_{\alpha}, \delta, \mu\right)$ следует из леммы 3.1 . Пусть $(P, \tau) \in M\left(g, 0,0, k_{\alpha}, \delta, \mu\right)$. Занумеруем овалы $c_{1}, \ldots, c_{k}\left(k=k_{0}+k_{1}\right)$ кривой $\left(P^{\#}, \tau^{\#}\right)$ так, чтобы

$$
\omega\left(c_{i}\right)= \begin{cases}0 & \text { при } i \leqslant n_{0}, \\ 1 & \text { при } i>n_{0},\end{cases}
$$

где $\omega=\omega_{1}(P)=\omega_{2}(P)$. Согласно [3], их можно дополнить непересекающимися псевдоовалами $c_{i}(k<i \leqslant n)$ так, чтобы множество $c_{1}, \ldots, c_{n}$ разбивало $P^{\#}$ на две компоненты связности. Наделим контуры $c_{1}, \ldots, c_{n-1}$ спинорной ориентацией. Разрежем $P$ по контурам $c_{1}, \ldots, c_{n-1}$. На полученной поверхности $Q$ инволюция $\tau^{\#}$ действует без неподвижных точек. Следовательно, псевдоовал $c_{n} \subset Q$ можно выбрать так, чтобы ориентация компоненты связности $P_{1}$ поверхности $Q \backslash c_{n}$ индуцировала на $c_{1}, \ldots c_{n-1}$ спинорную ориентацию.

Согласно [3], $\left(P^{\#}, \tau^{\#}\right)=\left(H^{\#} / \Gamma^{*}, \widetilde{\Gamma}^{*} / \Gamma^{*}\right)$, где $\Gamma^{*} \subset \widehat{\operatorname{Aut}}\left(H^{\#}\right)$ порождена множеством $t^{*}=\left\{A_{i}^{*}, B_{i}^{*}\left(i=1, \ldots, g_{0}\right), C_{j}^{*}(j=1, \ldots, n)\right\} \in T\left(g_{0}, n_{\alpha}, 0\right)$ и отображениями $S_{C_{j}^{*}}^{\#}(j \leqslant k), \widetilde{S}_{C_{j}}^{\#}(j>k)$, где сдвиги $C_{j}^{*}$ отвечают контурам $c_{j}$ и ориентация $c_{1}$ индуцирована $C_{1}$.

Выберем $\widetilde{\Gamma} \subset \widetilde{\operatorname{Aut}}(H)$ так, чтобы $(P, \tau)=(H / \Gamma, \widetilde{\Gamma} / \Gamma)$ и $\widetilde{\Gamma}^{\#}=\widetilde{\Gamma}^{*}$. Положим $t=(\#)^{-1}\left(t^{*}\right) \subset \Gamma$. Заменяя $t$ на сопряженный, можно считать, что $t=\left\{A_{i}, B_{i}\left(i=1, \ldots, g_{0}\right), C_{j}(j=1, \ldots, n)\right\}$ удовлетворяет условиям 1$\left.)-4\right)$ разд. 2 и $S_{C_{1}}^{0 \mu}$ (или $\widetilde{S}_{C_{1}}^{0 \mu}$, если $k=0$ ) принадлежит $\widetilde{\Gamma}$. Согласно [13], $A_{i}^{*}, B_{i}^{*}$ $(i=1, \ldots, g)$ можно выбрать таким образом, чтобы выполнялись условия 5$)$, т. е. $t \subset T\left(g_{0}, n_{\alpha}, \eta\right)$. В силу выбора ориентации на $c_{i}$ имеют место включения $S_{C_{i}}^{0 \mu} \in \widetilde{\Gamma}$ при $i \leqslant k$ и $\widetilde{S}_{C_{i}}^{0 \mu} \in \widetilde{\Gamma}$ при $k<i<n$. Согласно лемме 3.1, $\widetilde{S}_{C_{n}}^{\delta \mu} \in \widetilde{\Gamma}$ и, следовательно, $\Phi(t)=(P, \tau)$.

Теорема 3.1. Пространство модулей $M(g, \varepsilon, 0)$ вещественных алгебраических суперкривых $(P, \tau)$ c $N=2$ рода $g$, удовлетворяюших условию $\omega_{1}(P)=\omega_{2}(P)$, распадается на компоненты связности $M\left(g, 0,0, k_{\alpha}, \delta, \mu\right)$, $M\left(g, 1, k_{\alpha}^{\gamma}, \eta, \mu\right)$, где $\alpha, \gamma, \delta, \mu \in \mathbb{Z}_{2}, 0 \leqslant k_{0}+k_{1} \leqslant g, 1 \leqslant \sum_{\alpha, \gamma} k_{\alpha}^{\gamma} \leqslant g+1$, $\sum_{\alpha, \gamma} k_{\alpha}^{\gamma} \equiv g+1(\bmod 2), k_{0} \equiv g+1(\bmod 2), k_{0}^{0}+k_{0}^{1} \equiv g+1(\bmod 2) u \eta=0$ при $k_{1}^{0}+k_{1}^{1}>0$. Среди них совпадают лишь компоненты $M\left(g, 1,0, k_{\alpha}^{\gamma}, \eta, \mu\right)$ u $M\left(g, 1,0, k_{\alpha}^{1-\gamma}, \eta, \mu\right)$. Каждая из компонент $M(\chi)$ имеет вид $\mathbb{R}^{(4 g-3 \mid 4 g-4)} / \operatorname{Mod}(\chi)$, где $\operatorname{Mod}(\chi)$ дискретно действует на $\mathbb{R}^{(4 g-3 \mid 4 g-4)}$.

ДокаЗАТЕЛЬСтво. Согласно теореме 2.1 и леммам 3.2 и 3.3 , множество $M(g, \varepsilon, 0)$ распадается на множества $M(\chi)=\Phi\left(T\left(g_{0}, n_{\alpha}, \varepsilon\right)\right)$, где или $\chi=$ $\left(g, 0,0, k_{\alpha}, \delta, \mu\right)$ и $\Phi=\Phi\left(k_{\alpha}, \delta, \mu\right)$, или $\chi=\left(g, 1, k_{\alpha}^{\gamma}, \eta, \mu\right)$ и $\Phi=\Phi\left(k_{\alpha}^{\gamma}, \mu\right)$. Согласно [13], множество $T=T\left(g_{0}, n_{\alpha}, \varepsilon\right)$ естественным образом взаимно однозначно параметризуется множеством $\mathbb{R}^{\left(8 g_{0}+4 n-7 \mid 8 g_{0}+4 n-8\right)}=\mathbb{R}^{(4 g-3 \mid 4 g-4)}$. По построению $T$ можно рассматривать как множество пар $(\widetilde{\Gamma}, \tilde{t})$, где $\tilde{t}-$ указанный в леммах 3.2 и 3.3 базис группы $\widetilde{\Gamma}$ и $(H / \Gamma, \widetilde{\Gamma} / \Gamma) \in \Phi(t) ;$ здесь 
$\Gamma=\widetilde{\Gamma} \cap \operatorname{Aut}(H), t=\tilde{t} \cap \Gamma$. Выберем одну из пар $\left(\widetilde{\Gamma}_{0}, \tilde{t}_{0}\right) \in T$. Для любого $\varphi \in \operatorname{Aut}\left(\widetilde{\Gamma}_{0}\right)$ существует $F \in \operatorname{Aut}(H)$ и $\tilde{t}_{1} \in T$, такие, что $\varphi\left(\tilde{t}_{0}\right)=F \tilde{t}_{1} F^{-1}$. Условия нормировки 1$), 2$ ) определяют $t_{1}$ однозначно с точностью до сопряжения элементами группы $G \cong \mathbb{Z}_{2}^{2}$, порожденной инволюциями $\left(z \mid \theta_{1}, \theta_{2}\right) \mapsto$ $\left(z \mid-\theta_{1},-\theta_{2}\right)$ и $\left(z \mid \theta_{1}, \theta_{2}\right) \mapsto\left(z \mid \theta_{2}, \theta_{1}\right)$. Естественное соответствие между базисами $\tilde{t}$ и $\tilde{t}_{0}$ порождает действие $\operatorname{Aut}\left(\Gamma_{0}\right) / \operatorname{Aut}\left(\Gamma_{0}\right) \cap \operatorname{Aut}(H)$ на $T / G$. Таким образом, $\operatorname{Aut}\left(\Gamma_{0}\right) / \operatorname{Aut}\left(\Gamma_{0}\right) \cap \operatorname{Aut}(H)$ действует на $T / G$ и орбита этого действия совпадает с $M(\chi)$. Согласно [3], это действие дискретно.

\section{§4. Параметризация компонент связности пространства модулей кривых $(P, \tau)$, удовлетворяющих условию $\omega_{1}(P) \neq \omega_{2}(P)$}

1. Пусть $\omega_{1} \neq \omega_{2}$. Определенное в $\S 2$ двулистное накрытие $\varphi_{\#}: P_{*}^{\#} \rightarrow P^{\#}$ переводит овалы и псевдоовалы кривых $\left(P_{*}^{\#}, \tau_{i}^{\#}\right)$ в овалы и псевдоовалы кривой $\left(P^{\#}, \tau^{\#}\right)$. Обозначим через $Q_{i}$ множество псевдоовалов кривой $\left(P^{\#}, \tau^{\#}\right)$ вида $\varphi_{*}(c)$, где $c$ - псевдоовал кривой $\left(P_{*}^{\#}, \tau_{i}^{\#}\right)$.

Лемма $4.1[6,9]$. Овальь кривой $\left(P^{\#}, \tau^{\#}\right)$ можно дополнить попарно непересекаюшимися псевдоовалами $q_{1}, \ldots, q_{m} \subset Q_{1} \cup Q_{2}$ так, чтобы: (1) совокупность овалов и псевдоовалов $q_{1}, \ldots, q_{m}$ разбивала $P^{\#}$ на две компоненты связности; (2) каждое из множеств $Q_{i}$ содержало не более двух псевдоовалов; (3) если среди овалов есть такой овал с, ито $\varphi_{*}^{-1}(c)-$ свлзное множество, то все псевдооваль принадлежат $Q_{1} ;(4)$ если для каждого овала с множество $\varphi_{*}^{-1}(c)$ несвлзно $и Q_{i}=\varnothing$, mо $\varepsilon\left(P_{*}^{\#}, \tau_{i}\right)=1$.

2. Введем множество $T\left(g_{0}, n_{\alpha \beta}, \eta_{i}\right)$ (где $\alpha, \beta, i, \eta_{i} \in \mathbb{Z}_{2}, n=\sum_{\alpha, \beta \in \mathbb{Z}_{2}} n_{\alpha \beta}$ $>0, n_{10}+n_{11} \equiv n_{01}+n_{11} \equiv 0(\bmod 2), \eta_{1}=0$ при $n_{10}+n_{11}>0, \eta_{2}=0$ при $\left.n_{01}+n_{11}>0\right)$, параметризующее компоненты связности множества суперкривых $(P, \tau)$ рода $g=2 g_{0}+n-1$, таких, что существует овал $c \subset P^{\tau}$ со свойством $\omega_{1}(c) \neq \omega_{2}(c)$.

Оно состоит из всех наборов $t=\left\{A_{i}, B_{i}\left(i=1, \ldots, g_{0}\right), C_{j}(j=1, \ldots, n)\right\} \subset$ $(\operatorname{Aut}(H))^{g+1}$, таких, что

1) $C_{1}\left(Z \mid \theta_{1}, \theta_{2}\right)=\left(\lambda z \mid h^{1} \theta_{2}, h^{2} \theta_{1}\right)$, где $\lambda^{\#}>1$;

2) $C_{n}(1 \mid 0,0)=(1 \mid 0,0)$, если $n>1$, и $A_{1}(1 \mid 0,0)=(1 \mid 0,0)$, если $n=1$, причем $1 \in \mathbb{R}$ - притягивающая точка для $C_{1}^{\#}$ (соответственно для $A_{1}^{\#}$ );

3) $t$ порождает суперфуксову группу $\Gamma$ с определяющим соотношением

$$
\prod_{i=1}^{g_{0}}\left[A_{i}, B_{i}\right] \prod_{j=1}^{n} C_{j}=1,
$$

состоящую из гиперболических сдвигов;

4) на поверхности $P^{\#}=H^{\#} / \Gamma^{\#}$ рода $g_{0}$ с $n$ дырами сдвигам $C_{i}$ отвечают простые контуры $c_{i}(i \in I=\{1, \ldots, n\})$, охватывающие ровно одну дыру; при этом множество $I_{\alpha \beta}=\left\{i \in I, \Omega_{1}\left(C_{i}\right)=\alpha, \Omega_{2}\left(C_{i}\right)=\beta\right\} \quad\left(\right.$ где $\Omega_{i}=\Omega_{i}(\Gamma)$ ) содержит $n_{\alpha \beta}$ элементов и задает разбиение множества $I$, удовлетворяющее условию $I_{01}<I_{10}<I_{00}<I_{11}$;

5) $\Omega_{j}\left(A_{i}\right)=\Omega_{j}\left(B_{i}\right)=0$ при $i>1$ и $\Omega_{j}\left(A_{1}\right)=\Omega_{j}\left(B_{1}\right)=\eta_{j}$. 
Лемма 4.2. Пусть $\sum_{\gamma, \mu \in \mathbb{Z}_{2}} k_{\alpha \beta}^{\gamma \mu}=n_{\alpha \beta}$. Разобьем множество $I_{\alpha \beta}$ на состояшие из $k_{\alpha \beta}^{\gamma \mu}$ элементов подмножества $I_{\alpha \beta}^{\gamma \mu}\left(\gamma, \mu \in \mathbb{Z}_{2}\right)$ так, итобь $I_{\alpha \beta}^{00}<I_{\alpha \beta}^{10}<I_{\alpha \beta}^{01}<I_{\alpha \beta}^{11}$. Рассмотрим отображение $\Phi=\Phi\left(k_{\alpha \beta}^{\gamma \mu}\right)$, сопоставляющее базису $t=\left\{A_{i}, B_{i}\left(i=1, \ldots, g_{0}\right), C_{j}(j=1, \ldots, n)\right\} \in T\left(g_{0}, n_{\alpha \beta}, \eta_{i}\right)$ кривую $(P, \tau)=(H / \Gamma, \widetilde{\Gamma} / \Gamma)$, где группа $\widetilde{\Gamma}$ порождена $t$ и инволюииями $S_{C_{i}}^{\gamma_{i} \mu_{i}}$, такими, ито $\gamma_{i}=\gamma, \mu_{i}=\mu$, если $i \in I_{\alpha \beta}^{\gamma \mu}$. Тогда $\Phi\left(T\left(g_{0}, n_{\alpha \beta}, \eta_{i}\right)\right)=$ $M\left(g, 1,1, k_{\alpha \beta}^{\gamma \mu}, \eta_{i}\right)$.

ДоказАтельство. Утверждение $\Phi\left(T\left(g_{0}, n_{\alpha \beta}, \eta_{i}\right)\right) \subset M\left(g, 1,1, n_{\alpha \beta}^{\gamma \mu}, \eta_{i}\right)$ следует из леммы 3.1. Пусть $(P, \tau) \in M\left(g, 1,1, n_{\alpha \beta}^{\gamma \mu}, \eta_{i}\right)$. Занумеруем овалы $c_{1}, \ldots, c_{n}$ так, чтобы $\omega_{1}\left(c_{i}\right)=\alpha, \omega_{2}\left(c_{i}\right)=\beta, \xi^{\omega_{1}}\left(c_{1}, c_{i}\right)=\gamma$ и $\xi^{\omega_{2}}\left(c_{1}, c_{i}\right)=$ $\gamma+\mu+1$ при $i \in I_{\alpha \beta}^{\gamma \mu}$. Дальнейшее доказательство совпадает с доказательством леммы 3.2 .

ЛЕмма 4.3. Пусть $n_{11}-2 \leqslant k_{11}^{0}+k_{11}^{1}<n_{11} u k_{\alpha \beta}^{0}+k_{\alpha \beta}^{1}=n_{\alpha \beta}$, если $(\alpha, \beta) \neq(1,1)$. При $(\alpha, \beta) \neq(1,1)$ разобьем множества $I_{\alpha \beta}$ на состоящие из $k_{\alpha \beta}^{\mu}$ элементов подмножества $I_{\alpha \beta}^{\mu}$ так, чтобь $I_{\alpha \beta}^{0}<I_{\alpha \beta}^{1}$. Разобьем $I_{11}$ на подмножества $I_{11}^{0}<I_{11}^{1}<I_{11}^{*}$ так, чтобь $I_{11}^{\mu}$ содержало $k_{11}^{\mu}$ элементов при $\mu \in \mathbb{Z}_{2}$. Рассмотрим отображение $\Phi=\Phi\left(k_{\alpha \beta}^{\mu}, \delta_{i}\right)\left(i, \delta_{i} \in \mathbb{Z}_{2}\right)$, coпоставляюшее набору $t=\left\{A_{i}, B_{i}\left(i=1, \ldots, g_{0}\right), C_{j}(j=1, \ldots, n)\right\} \in I\left(g_{0}, n_{\alpha \beta}, \eta_{i}\right)$ кривую $(P, \tau)=(H / \Gamma, \widetilde{\Gamma} / \Gamma)$, где группа $\widetilde{\Gamma}$ порождена набором $t$ и инволюииями $S_{C_{i}}^{0 \mu_{i}}\left(\right.$ дде $\left.i \in I_{\alpha \beta}^{\mu} \neq I_{11}^{*}, \mu_{i}=\mu\right), \widetilde{S}_{C_{i}}^{\sigma_{i} \mu_{i}}\left(\right.$ дде $i \in I_{11}^{*}, \sigma_{i}=0, \mu_{i}=\mu_{1}$, если $\left.i<n, \sigma_{n}=\delta_{1}, \sigma_{n}+\mu_{n}=\delta_{2}\right)$. Тогда $\Phi\left(T\left(g_{0}, n_{\alpha \beta}, \eta_{i}\right)\right)=T\left(g, 0,1, k_{\alpha \beta}^{\mu}, \delta_{i}, \rho_{i}\right)$, где $\rho_{1}=\rho_{2}=0$.

ДокаЗАтельство. Утверждение $\Phi\left(T\left(g_{0}, n_{\alpha \beta}, \eta_{i}\right)\right) \subset M\left(g, 0,1, k_{\alpha \beta}^{\mu}, \delta_{i}, \rho_{i}\right)$ следует из леммы 3.1. Пусть $(P, \tau) \in M\left(g, 0,1, k_{\alpha \beta}^{\mu}, \delta_{i}, \rho_{i}=0\right)$. Занумеруем овалы $c_{1}, \ldots, c_{k}\left(k=\sum_{\alpha, \beta, \mu \in \mathbb{Z}_{2}} k_{\alpha \beta}^{\mu}\right)$ кривой $\left(P^{\#}, \tau^{\#}\right)$ так, чтобы $\omega_{1}\left(c_{i}\right)=\alpha$, $\omega_{2}\left(c_{i}\right)=\beta, \mu\left(c_{i}\right)=\mu$ при $i \in I_{\alpha \beta}^{\mu}$. Согласно лемме 4.1, их можно дополнить псевдоовалами $q_{i}(1 \leqslant i \leqslant n-k \leqslant 2)$, удовлетворяющими условиям леммы 4.1. При $n=k+2$ положим $c_{n-1}=q_{1}$. Повторяя рассуждения леммы 3.3 , находим псевдоовал $c_{n}$, такой, чтобы контуры $c_{1}, \ldots, c_{n}$ разбивали $P^{\#}$ на две компоненты, одна из которых индуцирует спинорную ориентацию на $c_{1}, \ldots, c_{n-1}$. Завершающая часть доказательства совпадает с соответствующей частью доказательства леммы 3.3 .

3. Введем множество $T\left(g_{0}, n_{\alpha}, \eta_{i}\right)$ (где $\alpha, i, \eta_{i} \in \mathbb{Z}_{2}, n=n_{0}+n_{1}>0$, $n_{1} \equiv 0(\bmod 2), \eta_{1}=\eta_{2}=0$ при $\left.n_{1}>0\right)$, параметризующее компоненты связности множества суперкривых $(P, \tau)$ рода $g=2 g_{0}+n-1$, таких, что $\omega_{1} \neq \omega_{2}$, но $\omega_{1}(c)=\omega_{2}(c)$ для любого овала $c \subset P^{\tau}$.

Оно состоит из всех наборов $t=\left\{A_{i}, B_{i}\left(i=1, \ldots, g_{0}\right), C_{j}(j=1, \ldots, n)\right\} \subset$ $(\operatorname{Aut}(H))^{g+1}$, таких, что

1) $C_{n}\left(Z \mid \theta_{1}, \theta_{2}\right)=\left(\lambda z \mid h^{1} \theta_{1}, h^{2} \theta_{2}\right)$, где $\lambda^{\#}>1$;

2) $C_{1}(1 \mid 0,0)=(1 \mid 0,0)$, если $n>1$, и $A_{1}(1 \mid 0,0)=(1 \mid 0,0)$, если $n=1$, причем $1 \in \mathbb{R}$ - притягивающая точка для $C_{1}^{\#}$ (соответственно для $A_{1}^{\#}$ ); 
3) $t$ порождает суперфуксову группу $\Gamma$ с определяющим соотношением

$$
\prod_{i=1}^{g_{0}}\left[A_{i} B_{i}\right] \prod_{j=1}^{n} C_{j}=1,
$$

состоящую из гиперболических сдвигов;

4) на поверхности $P^{\#}=H^{\#} / \Gamma^{\#}$ рода $g_{0}$ с $n$ дырами сдвигам $C_{i}$ отвечают простые контуры $c_{i}(i \in I=\{1, \ldots, n\})$, охватывающие ровно одну дыру, причем $\Omega_{j}\left(C_{i}\right)=\alpha$, если $i \in I_{\alpha}$, где $I_{0}=\left\{i \in I: i \leqslant n_{0}\right\}, I_{1}=\left\{i \in I: i>n_{0}\right\}$;

5) а) $\Omega_{1}\left(A_{i}\right)=\Omega_{1}\left(B_{i}\right)=0$ при $i>1$ и $\Omega_{1}\left(A_{1}\right)=\Omega_{1}\left(B_{1}\right)=\eta_{1}$,

b) $\Omega_{2}\left(A_{i}\right)=\Omega_{2}\left(B_{i}\right)=0$ при $i>2$ и $\Omega_{2}\left(A_{2}\right)=\Omega_{2}\left(B_{2}\right)=\eta_{1} \cdot \eta_{2}$,

c) $\Omega_{2}\left(B_{1}\right)=0, \Omega_{2}\left(A_{1}\right)=\eta_{2}$.

Повторяя доказательство леммы 4.2, получаем такой результат:

Лемма 4.4. Пусть $\sum_{\gamma, \mu} k_{\alpha \alpha}^{\gamma \mu}=n_{\alpha}$. Разобьем множество $I_{\alpha}$ на состоящие из $k_{\alpha \alpha}^{\gamma \mu}$ элементов подмножества $I_{\alpha}^{\gamma \mu}\left(\gamma, \mu \in \mathbb{Z}_{2}\right)$ так, чтобь $I_{\alpha}^{00}<$ $I_{\alpha}^{01}<I_{\alpha}^{10}<I_{\alpha}^{11}$. Рассмотрим отображение $\Phi=\Phi\left(k_{\alpha \alpha}^{\gamma \mu}\right)$, сопоставляюшее набору $t=\left\{A_{i}, B_{i}\left(i=1, \ldots, g_{0}\right), C_{j}(j=1, \ldots, n)\right\} \in T\left(g_{0}, n_{\alpha}, \eta_{i}\right)$ кривую $(P, \tau)=(H / \Gamma, \widetilde{\Gamma} / \Gamma)$, аде аруппа $\widetilde{\Gamma}$ порождена набором $t$ u инволючиями $S_{C_{i}}^{\gamma_{i} \mu_{i}}$, такими, что $\gamma_{i}=\gamma, \mu_{i}=\mu$, если $i \in I_{\alpha}^{\gamma \mu}$. Tогда $\Phi\left(T\left(g_{o}, n_{\alpha}, \eta_{i}\right)\right)=$ $M\left(g, 1,1, k_{\alpha \beta}^{\gamma \mu}, \eta_{i}\right)$, где $k_{01}^{\gamma \mu}=k_{10}^{\gamma \mu}=0$.

Небольшая модификация доказательства леммы 4.3 дает следующую лемму:

Лемма 4.5. Пусть $n_{1}-4 \leqslant k_{11}^{0}+k_{11}^{1}<n_{1} u k_{00}^{0}+k_{00}^{1}=n_{0}$. Разобьем множество $I_{0}$ на состояшие из $k_{00}^{\mu}$ элементов подмножества $I_{0}^{\mu}\left(\mu \in \mathbb{Z}_{2}\right)$ так, чтобьи $I_{0}^{0}<I_{0}^{1}$. Разобьем $I_{1}$ на подмножества $I_{1}^{0}<I_{1}^{1}<J^{0}<J^{1}$ maк, чтобьи $I_{1}^{\mu}$ содержало $k_{11}^{\mu}$ элементов при $\mu \in \mathbb{Z}_{2}$ u $J^{\mu}$ содержало $0 \leqslant m^{\mu} \leqslant 2$ элементов. Рассмотрим отображение $\Phi=\Phi\left(k_{\alpha \alpha}^{\mu}, \delta_{1}, \rho_{i}\right)\left(i, \delta_{1}, \rho_{i} \in \mathbb{Z}_{2}\right)$, сопоставляюшее набору $t=\left\{A_{i}, B_{i}\left(i=1, \ldots, g_{0}\right), C_{j}(j=1, \ldots, n)\right\} \in$ $T\left(g_{0}, n_{\alpha}, \eta_{i}\right)$ кривую $(P, \tau)=(H / \Gamma, \widetilde{\Gamma} / \Gamma)$, әде әруппа $\widetilde{\Gamma}$ порождена набором $t$ u инволюииями $S_{C_{i}}^{0 \mu_{i}}\left(\right.$ әде $\left.i \in I_{\alpha}^{\mu}, \mu_{i}=\mu\right), \widetilde{S}_{C_{i}}^{\sigma_{i} \mu_{i}}\left(\right.$ әде $i \in J^{\mu}, \mu_{i}=\mu$; $\sigma_{i}=0$, если $\left.i<n, \sigma_{n}=\delta_{1}\right)$. Тогда $\Phi\left(T\left(g_{o}, n_{\alpha}, 0\right)\right)=M\left(g, 0,1, k_{\alpha \beta}^{\mu}, \delta_{i}, \rho_{i}\right)$, где $k_{01}^{\mu}=k_{10}^{\mu}=0, \rho_{i}=1$, если и только если $m^{i}=0, \delta_{2} \equiv m^{0}+k_{11}^{0}(\bmod 2)$, если $J^{1} \neq \varnothing$, и $\delta_{2} \equiv k_{11}^{1}(\bmod 2)$, если $J^{1}=\varnothing$.

ТеоремА 4.1. Пространство модулей $M(g, \varepsilon, 1)$ вещественных алгебраических суперкривых $(P, \tau)$ с $N=2$ рода $g$, удовлетворяюших условию $\omega_{1}(P)$ $\neq \omega_{2}(P)$, распадается на компоненты связности вида $M\left(g, 0,1, k_{\alpha \beta}^{\mu}, \delta_{i}, \rho_{i}\right)$, $M\left(g, 1,1, k_{\alpha \beta}^{\gamma \mu}, \eta_{i}\right)$, әде $\alpha, \beta, \gamma, \mu, i, \delta_{i}, \rho_{i}, \eta_{i} \in \mathbb{Z}_{2}, 0 \leqslant \sum_{\alpha, \beta, \mu} k_{\alpha \beta}^{\mu} \leqslant g, 1 \leqslant$ $\sum_{\alpha, \beta, \gamma, \mu} k_{\alpha \beta}^{\gamma \mu} \leqslant g+1, \sum_{\alpha, \beta, \gamma, \mu} k_{\alpha \beta}^{\gamma \mu} \equiv g+1(\bmod 2), \sum_{\mu, \beta} k_{0 \beta}^{\mu} \equiv \sum_{\mu, \alpha} k_{\alpha 0}^{\mu} \equiv$ $\sum_{\gamma, \mu, \beta} k_{0 \beta}^{\gamma \mu} \equiv \sum_{\gamma, \mu, \alpha} k_{\alpha 0}^{\gamma \mu} \equiv g+1(\bmod 2), \rho_{1}+\rho_{2}<2, \rho_{1}=\rho_{2}=0$ nрu $k_{01}^{0}+$ $k_{01}^{1}+k_{10}^{0}+k_{10}^{1}>0, \eta_{1}=0$ nрu $\sum_{\alpha, \gamma, \mu} k_{1 \beta}^{\gamma \mu}>0 u \eta_{2}=0$ nрu $\sum k_{\alpha 1}^{\gamma, \mu}>0$. Cредu них совпадают лишь множества $M\left(g, 1,1, k_{\alpha \beta}^{\gamma \mu}, \eta_{i}\right) u M\left(g, 1,1, k_{\alpha \beta}^{1-\gamma \mu}, \eta_{i}\right)$. Каждая компонента $M(\chi)$ имеет вид $\mathbb{R}^{(4 g-4 \mid 4 g-4)} / \operatorname{Mod}(\chi)$, әде $\operatorname{Mod}(\chi)$ дискретно действует на $\mathbb{R}^{(4 g-4 \mid 4 g-4)}$.

ДокАЗАтЕЛЬство. Согласно теореме 2.1 и леммам 4.2-4.5, множество $M(g, \varepsilon, 1)$ распадается на подмножества $M(\chi)=\Phi\left(T\left(g_{0}, n_{\alpha \beta}, \eta_{i}\right)\right)$. Согласно 
[13], множество $T=T\left(g_{0}, n_{\alpha \beta}, \eta_{i}\right)$ естественным образом взаимно однозначно параметризуется множеством $\mathbb{R}^{\left(8 g_{0}+4 n-7 \mid 8 g_{0}+4 n-8\right)}=\mathbb{R}^{(4 g-3 \mid 4 g-4)}$. По построению $T$ можно рассматривать как пару $(\widetilde{\Gamma}, \tilde{t})$, где $\tilde{t}$ - указанный в леммах 4.2-4.5 базис группы $\widetilde{\Gamma}$ и $(H / \Gamma, \widetilde{\Gamma} / \Gamma) \in \Phi(t) ;$ здесь $\Gamma=\widetilde{\Gamma} \cap \operatorname{Aut}(H), t=$ $\tilde{t} \cap \Gamma$. Выберем одну из пар $\left(\widetilde{\Gamma}_{0}, \tilde{t}_{0}\right) \in T$. Естественное соответствие между базисами $\tilde{t}$ и $\tilde{t}_{0}$ индуцирует действие группы $\operatorname{Aut}\left(\widetilde{\Gamma}_{0}\right)$, переводящее $(\widetilde{\Gamma}, \tilde{t})$ в $\left(F \widetilde{\Gamma}^{1} F, F \tilde{t}^{1} F^{-1}\right)$ для некоторых $F \in \operatorname{Aut}(H)$ и $\left(\widetilde{\Gamma}^{1}, \tilde{t}^{1}\right) \in T$. Условия 1$\left.), 2\right)$ однозначно определяют $\tilde{t}^{1}$ с точностью до сопряжения элементом группы $G$, порожденной преобразованиями вида $\left(z \mid \theta_{1}, \theta_{2}\right) \mapsto\left(z \mid \mu \theta_{i}, \mu^{-1} \theta_{3-i}\right),\left(z \mid \theta_{1}, \theta_{2}\right) \mapsto$ $\left(z \mid-\theta_{1},-\theta_{2}\right),\left(z \mid \theta_{1}, \theta_{2}\right) \mapsto\left(z \mid \theta_{2}, \theta_{1}\right)$. Согласно [13], $T / G \cong \mathbb{R}^{(4 g-4 \mid 4 g-4)} / \mathbb{Z}_{2}^{2}$.

\section{ЛиТЕРАТУРА}

1. Баранов M. А., Швари А. С. О многопетлевом вкладе в теории струны. Письма в ЖКЭТФ, 42, №8, 340-342 (1985).

2. Манин Ю. И. Супералгебраические кривые и квантовые струны. Труды МИАН СССР, 183, 126-138 (1990).

3. Натанзон C. М. Модули вещественных алгебраических кривых. Труды Моск. матем. о-ва, 37, 219-253 (1978).

4. Натанзон С. М. Пространство модулей римановых суперповерхностей. Матем. заметки, 45, №4, 111-116 (1989).

5. Натанзон С. М. Клейновы суперповерхности. Матем. заметки, 48, №2, 72-82 (1990).

6. Натанзон С. М. Клейновы поверхности. УМН, 45, вып. 6, 47-90 (1990).

7. Натанзон C. М. Классификация пар функций Арфа на ориентируемых и неориентируемых поверхностях. Функц. анализ и его прил., 28, вып. 3, 35-46 (1994).

8. Atiyah M. Riemann surfaces and spin structures. Ann. Sci. École Norm. Sup. (4), 4, No. 1, 47-62 (1971).

9. Bujalance E., Costa A. F., Natanzon S. M., Singerman D. Involutions of compact Klein surfaces. Math. Z., 211, 461-478 (1992).

10. Friedan $D$. Notes on string theory and two dimensional conformal field theory. Proc. Workshop on Unified String Theories (Institute for Theoretical Physics, Santa Barbara) 1985.

11. Gepner D. Space-time supersymmetry in compactified string theory and superconformal models. Nuclear Phys B, 296, 757-779 (1988).

12. Mumford D. Theta characteristics of an algebraic curve. Ann. Sci. Ecole. Norm. Sup. (4), 4, No. 2, 181-192 (1971),

13. Natanzon S. M. Moduli spaces of Riemann $N=1$ and $N=2$ supersurfaces. J. Geom. Phys., 12, 35-54 (1993).

14. Natanzon S. M. Moduli spaces of Riemann and Klein supersurfaces. Development of Modern Mathematics, Moscow School, eds. V. Arnold and M. Monastyrsky, Chapman and Hall, England, 1993, pp. 100-130

Независимый московский университет

Поступило в редакцию 30 октября 1995 г. 